\title{
Responsabilidad social corporativa. Una visión desde la teoría económica*
}

\author{
Aleix Calveras \\ Universitat de les Illes Balears \\ Juanjo Ganuza \\ Universitat Pompeu Fabra
}

\section{Resumen}

El objetivo de este trabajo es discutir y analizar el concepto de la responsabilidad social corporativa (RSC) desde la teoría económica. Para ello usamos un modelo sencillo que nos permite clarificar algunos aspectos del debate que rodea la RSC. Respecto a la motivación empresarial para adoptar políticas RSC, nuestra opinión es que ésta se origina en una demanda del mercado, lo que implica que no tiene que estar en contradicción con la rentabilidad empresarial, más bien al contrario. Así, la RSC consiste en adoptar una visión a largo plazo de la maximización del beneficio empresarial basada en gran medida en mantener una estrategia de cooperación con los distintos stakeholders de la empresa. Otro aspecto clave de la RSC es su relación e interacción con la intervención y regulación pública. Desde nuestro punto de vista, el papel de la RSC será importante allí donde la regulación pública no puede llegar debido a, por ejemplo, restricciones de información a las que se enfrenta el sector público. Finalmente, analizamos también el papel y las características del activismo desarrollado por algunos grupos de consumidores (mediante el posible lanzamiento de boicots a las empresas no responsables socialmente).

Palabras clave: stakeholders, regulación, activismo de los consumidores.

Clasificación JEL: D21, L14, L51, M21.

\begin{abstract}
The objective of this paper is to analyze the concept of corporate social responsibility (CSR) from the perspective of economic theory. To do so, we use a simple model which allows us to clarify some issues. To our view, CSR is demand driven, which implies that it does not have to be in contradiction with firm's profitability, rather the opposite. Thus, a socially responsible strategy by a firm consists of maximizing profits on the long term, based upon a strategy of cooperation with all stakeholders of the firm. Another key aspect in relation to CSR is its interaction with public regulation. To our view, CSR is important in those areas where public regulation is not useful due to, for instance, informational restrictions that the public sector faces. Finally, we also analyze the role and characteristics of activism developed by some consumers.
\end{abstract}

Keywords: stakeholders, regulation, consumer activism.

JEL classification: D21, L14, L51, M21.

\section{Introducción}

Tradicionalmente, la visión económica de la empresa ha definido a ésta como a una organización cuyo objetivo es la maximización de beneficios. Este tipo de empresa, que denominaremos neoclásica, se preocupa por tanto por el bienestar - los 
beneficios de los propietarios de la empresa, los accionistas. En condiciones ideales el primer teorema de la economía del bienestar asegura que un sistema económico en el que las empresas maximizan el beneficio de sus propietarios (los accionistas) genera una asignación eficiente de los recursos.

Recientemente, sin embargo, ha ganado fuerza la visión de que la empresa debería tener un comportamiento Socialmente Responsable. Con este término calificaríamos a la empresa que no se preocupa solamente de la maximización del beneficio, sino que además tiene en cuenta el impacto de sus decisiones y estrategias sobre todos los interesados de la propia empresa, los stakeholders ${ }^{1}$ como son los trabajadores, los clientes, la comunidad local en la que la empresa actúa, etc, además de los propios accionistas. La justificación básica de esta postura desde la teoría económica se basa en que las condiciones para la aplicabilidad del primer teorema de la economía del bienestar no se cumplen en la realidad. En el mercado existen externalidades, información asimétrica entre los agentes, poder de mercado por parte de algunos de éstos, y todo esto implica que la asignación de mercado con agentes maximizadores de su beneficio privado no conducirá a una asignación eficiente de los recursos. Desde el enfoque de gestión empresarial Socialmente Responsable, la empresa a la hora de diseñar e implementar sus estrategias debe tener en cuenta los efectos externos de las mismas sobre todos los agentes sociales implicados en su actividad, los stakeholders.

El objetivo de este trabajo es hacer una revisión de la literatura sobre Responsabilidad Social Corporativa de las empresas, desde el ángulo de la teoría económica. No queremos hacer una revisión exhaustiva de la literatura ${ }^{2}$, si no revisar los conceptos claves de la RSC con un enfoque crítico, discutiendo los pros y los contras de este enfoque de la gestión empresarial. Además, nuestra revisión en gran parte no va a ser neutral. Aunque intentaremos reflejar los distintos puntos de vista sobre la cuestión, no rehuiremos tomar postura en algunos de los debates que revisaremos. En primer lugar, el trabajo clarifica lo que a nuestro juicio significa el concepto de responsabilidad social corporativa. De acuerdo con nuestra opinión, la RSC responde a una demanda del mercado, demanda que puede venir dada por distintos mecanismos y factores. Así, la RSC consiste en adoptar una serie de políticas que ponen el énfasis en el largo plazo más que en el corto plazo, y que favorecen unas políticas de cooperación con todos los stakeholders de la empresa (trabajadores, consumidores, etc.). Así, de hecho, y des de nuestra perspectiva, la RSC no está reñida con la maximización del beneficio empresarial (al menos a largo plazo). En este sentido, al final de la sección 2 discutimos una serie de políticas que frecuentemente reci-

${ }^{1}$ Dado que la gran parte de la literatura sobre responsabilidad social corporativa es en inglés, para no crear confusión con los términos, vamos a emplear la palabra «stakeholder» en lugar de grupo de interés, partícipe o afectado por la acción de la empresa.

${ }^{2}$ No obstante debemos decir que hasta donde nosotros sabemos, no existe mucha literatura sobre la RSC desde la teoría económica. Existen varias reseñas de la literatura académica de la RSC principalmente desde el 'management', ver RODRÍGUEZ (2002) y LA FUENTE et al. (2003). 
ben la etiqueta de socialmente responsables y que también se pueden justificar desde el enfoque puramente neoclásico de la maximización de beneficios. ${ }^{3}$

En la sección 2 del trabajo presentamos y discutimos el concepto de responsabilidad en un modelo sencillo en el que, debido a la posible presencia de una externalidad en la producción de un bien, existe espacio para el comportamiento socialmente responsable de la empresa. En este modelo están presentes dos tipos de stakeholders: por un lado los accionistas/propietarios de la empresa; y por el otro los consumidores que potencialmente sufren la externalidad. Observamos y analizamos cuál es el comportamiento de una empresa neoclásica maximizadora del beneficio, y cuál sería un comportamiento socialmente responsable.

Otro aspecto que consideramos de interés, y que analizamos dentro del modelo propuesto, es el papel que puede jugar el comportamiento activista de los consumidores para que las empresas neoclásicas tengan incentivos a comportarse de una forma socialmente responsable. En general, los consumidores no internalizan en sus decisiones de consumo el comportamiento de las empresas. Pero existen grupos de consumidores activistas que toman en consideración el comportamiento social de la empresa, a la hora de consumir. Este grupo de consumidores activistas puede lanzar campañas de boicot contra empresas con comportamientos sociales que juzguen deficientes. Estos consumidores activistas hacen que la demanda de la empresa sea sensible a su comportamiento social, dándole incentivos privados a adoptar políticas socialmente responsables.

En la sección 3, se realiza un sencillo análisis de bienestar, reflexionando sobre el comportamiento de los consumidores, tanto los que son activistas como los que no lo son. Se identifican dos factores determinantes en el lanzamiento de boicots de los consumidores a las empresas: (i) la información sobre el comportamiento real de las empresas, es decir, el saber efectivamente qué tipo de externalidades esta generando la empresa; y (ii) el comportamiento no oportunista de los consumidores. Las campañas de boicot adolecen del típico problema existente en las acciones colectivas, el problema del pasajero sin billete (o free-rider): cada consumidor prefiere que otro lleve a cabo la campaña (y asuma los costes de ésta) e induzca a la empresa a cambiar su estrategia.

En esta sección se analiza también otro aspecto clave de la RSC como es la intervención gubernamental, especialmente en dos aspectos: (i) la regulación, y (ii) la generación de información. La regulación es el mecanismo clásico para resolver los problemas de externalidades y, en general, de fallos de mercado. Por lo tanto, es necesario justificar la RSC como un mecanismo o un enfoque alternativo a la regu-

${ }^{3}$ Así, no adoptamos el punto de vista de Baron quién en la definción de las políticas RSC cuestiona la motivación real de las empresas para adoptar esas políticas. Según Baron, la RSC no depende sólo de las consecuencias de las acciones de la empresa sobre los stakeholders, hay que tener en cuenta la motivación real de la empresa. Así, unas políticas serían solamente socialmente responsables si van más allá de la maximización del beneficio empresarial, mientras que no lo serían si reportan una ganacia empresarial. 
lación. Nuestro punto de vista al respecto es que son mecanismos sustitutivos, y que la RSC adquiere más relevancia en entornos en los que por distintas causas la regulación o no existe o es poco efectiva. En tal caso sin embargo, el Sector Público aún puede jugar un papel relevante en lo que respecta a la generación y el control de la información relativa al comportamiento social de las empresas. El trabajo demuestra que el comportamiento de los consumidores activistas puede ser socialmente valioso, dado que ayuda a disciplinar el comportamiento de las empresas. Sin embargo, para que la presión de los consumidores sea efectiva, es necesario que estos tengan acceso a medidas fiables sobre el comportamiento social de las empresas. Es en este punto donde el sector público puede intervenir, no solamente exigiendo información veraz a las empresas, sino produciendo y seleccionando sus propios indicadores de RSC.

\section{RSC: un modelo}

En esta sección presentamos un modelo sencillo de responsabilidad social corporativa que nos servirá para discutir algunas de las ideas y conceptos que consideramos más importantes del enfoque de gestión basado en los stakeholders. En el modelo que proponemos hay solamente dos tipos de stakeholders: los accionistas/propietarios de la empresa y los potenciales consumidores (por ejemplo, no incluye trabajadores). Sin embargo, creemos que los conceptos e ideas que analizamos se pueden generalizar a empresas con un mayor número y diversidad de stakeholders.

En el mercado hay una empresa que actúa como un monopolio. La empresa tiene que elegir entre dos tipos de tecnologías mediante las cuales producir un bien: la tecnología limpia y la tecnología sucia. Producir con la tecnología limpia supone para la empresa un coste unitario de producción igual a $\mathrm{c}_{\mathrm{A}}$, mientras que el coste unitario de producción con la tecnología sucia es $\mathrm{c}_{\mathrm{B}}$, donde $\mathrm{B}$ representa una externalidad que surge en el proceso de producción. B puede representar tipos muy distintos de externalidades; de forma intuitiva, externalidades medioambientales generadas durante el proceso de producción (contaminación, del agua o aire, tala de arboles, etc.); también podría representar el hecho de contar con trabajo infantil durante el proceso de producción. En todo caso, es una externalidad que se genera al producir una unidad del bien y que recae sobre toda la sociedad, sobre los stakeholders de la empresa.

Para hacer el modelo interesante, suponemos que $c_{B}+B>c_{A}>c_{B}$. Es decir, el coste unitario de la tecnología sucia $\mathrm{c}_{\mathrm{B}}$ es menor que el coste unitario de la tecnología limpia $\mathrm{c}_{\mathrm{A}}$. Sin embargo, aunque el coste unitario de la tecnología sucia es menor, la tecnología limpia es socialmente eficiente debido a que no genera externalidades $\left(c_{B}+B>c_{A}\right)$.

En el mercado existe un continuo de consumidores que pueden consumir una unidad del bien producido por la empresa. Existen dos tipos de consumidores: una 
proporción · de activistas (A) y una proporción $1-\alpha$ de no activistas (NA). La función de utilidad de los activistas es

$$
u_{A}=v-p-\delta B
$$

y la función de utilidad de los no activistas es

$$
u_{N A}=v-p
$$

donde $\mathrm{v}$ es la valoración exógena de una unidad del bien, p es el precio del bien, B es la externalidad que se genera mediante la producción con la tecnología sucia, y $\delta=0$ si la tecnología es limpia, mientras que $\delta=1$ si la tecnología es sucia. Vemos pues que los consumidores no activistas no tienen en cuenta la tecnología (ni la externalidad que se genera) en la decisión de compra, mientras que los consumidores activistas sí que la tienen en cuenta: los activistas internalizan la externalidad en su decisión de consumo.

Suponemos también para simplificar que $c_{B}+B>v>c_{A}$. Esta hipótesis implicará que los consumidores activistas no comprarán el bien cuando éste sea producido con la tecnología sucia. Analicemos pues la decisión de la empresa respecto al tipo de tecnología que adopta. ${ }^{4}$

\section{Una empresa altruista}

Como benchmark, supongamos que la empresa maximiza sus beneficios, pero además internaliza las externalidades que su proceso de producción tiene sobre la sociedad; en otras palabras, en su función objetivo aparece también el bienestar de sus stakeholders..$^{5}$ Por lo tanto, la función de beneficios que maximiza es, en caso de utilizar la tecnología limpia, $\pi_{A}(L)=p-c_{A}$, y en caso de utilizar la tecnología sucia, $\pi_{A}(S)=p-c_{B}-B$, donde el subíndice A denota Altruista. En este caso, es muy fácil ver que la empresa no adoptará una tecnología sucia. Para que lo hiciera, el precio tendría que compensar la externalidad negativa, $p>c_{B}+B$, pero a este precio ningún consumidor compraría el bien con lo que la empresa no obtendría beneficios positivos.

${ }^{4}$ FEDDERSEN y GILLIGAN (2000) analizan un modelo similar en el que existen dos bienes, pero en el que no se analiza el proceso de fijación de precios.

${ }^{5}$ Estamos suponiendo que la empresa es parcialmente altruista, en el sentido de que internaliza las externalidades de su proceso de producción, pero no el excedente de los consumidores. En otras palabras, no es un planificador social y, tomando en consideración las externalidades que produce, no renuncia a maximizar sus beneficios pecuniarios. 
Si la empresa adopta la tecnología limpia, la función la función de utilidad de los consumidores activistas y no activistas coincide puesto que $\delta=0\left(u_{\mathrm{A}}=u_{N A}=v-p\right)$; entonces el precio que maximiza los beneficios de la empresa es $p=v$ y sus beneficios son $\pi_{A}(L)=v-c_{A}>0$. Por todo esto, si la empresa es altruista e internaliza las externalidades sobre sus stakeholders su elección será utilizar la tecnología limpia y, por lo tanto, mostrará un comportamiento socialmente responsable.

\section{Una empresa neoclásica}

Por empresa neoclásica entendemos una empresa tradicional que se preocupa de maximizar beneficios pero no de las externalidades que produce sobre el resto de la sociedad, sobre sus stakeholders. Por lo tanto, la función de beneficios que maximiza es, en caso de utilizar una tecnología limpia, $\pi_{N}(L)=p-c_{A}$, y en caso de utilizar una tecnología sucia, $\pi_{N}(S)=p-c_{B}$, donde el subíndice $\mathrm{N}$ denota Neoclásica. Como podemos comprobar, la función de beneficios de la empresa neoclásica y la de la empresa altruista coinciden cuando adoptan la tecnología limpia, por lo que su comportamiento y beneficios finales en tal caso son los mismos, $\pi_{N}(L)=\pi_{A}(L)=$ $v-c_{A}>0$.

Por otro lado, si la empresa adopta la tecnología sucia, los consumidores activistas no comprarán puesto que la función de utilidad de los activistas es $u_{A}=v-p-B$. En tal caso, solamente los consumidores no activistas compraran el bien y la empresa fija de forma óptima un precio $p=v$; y el beneficio de la empresa neoclásica cuando adopta la tecnología sucia es $\pi_{N}(S)=(1-\alpha)\left(v-c_{B}\right)$.

Así pues, la empresa adopta la tecnología limpia siempre y cuando

$$
\pi_{N}(L)=v-c_{A}>\pi_{N}(S)=(1-\alpha)\left(v-c_{B}\right)
$$

Con un cálculo sencillo, se comprueba que la empresa tradicional (o neoclásica) elige la tecnología limpia si y solo si

$$
\alpha>\frac{c_{A}-c_{B}}{v}
$$

Es decir, la empresa adopta la tecnología limpia solamente cuando hay una proporción lo suficientemente grande de consumidores activistas en el mercado, en cuyo caso la empresa tiene también un comportamiento socialmente responsable.

Como primeras conclusiones a este sencillo modelo obtenemos que:

1. La empresa altruista es socialmente responsable, internalizando la externalidad que su modo de producción genera en los consumidores, adoptando por lo tanto la tecnología limpia.

2. La empresa neoclásica, en cambio, en ausencia de consumidores activistas no 
presenta un comportamiento socialmente responsable, es decir, adopta la tecnología sucia.

3. Cuando existe un número suficientemente alto de consumidores activistas, éstos inducen a la empresa neoclásica a mostrar un comportamiento socialmente responsable, adoptando la tecnología limpia.

\section{Motivación y responsabilidad social corporativa}

En las secciones anteriores hemos comprobado como tanto la empresa altruista como la empresa neoclásica maximizadora de beneficios pueden adoptar políticas o estrategias que podemos denominar Socialmente Responsables. Sin embargo, su motivación es muy diferente: mientras la primera lo hace por que incorpora a su función objetivo el bienestar social (el impacto que tiene su actividad sobre los stakeholders), la empresa tradicional (o neoclásica) lo hace para no perder una parte del mercado, los consumidores que hemos denominado activistas.

¿Son ambas empresas socialmente responsables?, ¿deberíamos tener en cuenta la motivación de la empresa a la hora de denominarla socialmente responsable? Baron (2001) analiza estas preguntas y opina que, de cara a denominar una empresa Socialmente Responsable, se debe considerar no solamente su comportamiento, sino además su motivación. El criterio «moral» que defiende Baron consiste en denominar una empresa socialmente responsable solamente si existe una redistribución de excedente de los accionistas de la empresa a los stakeholders o, con otras palabras, si la acción socialmente responsable de la empresa no se puede explicar bajo la hipótesis de maximización de beneficios.

En nuestro modelo, siguiendo el criterio de Baron, si observamos que la empresa ha elegido la tecnología limpia sólo podemos concluir que es socialmente responsable si $\alpha>\frac{c_{A}-c_{B}}{v}$, es decir, si el número de consumidores activistas es demasiado pequeño como para que el miedo al potencial boicot justifique la elección de esa estrategia por parte de una empresa neoclásica o maximizadora. En caso contrario, aunque observásemos que la empresa usa tecnología limpia, esto no sería concluyente para denominarla Socialmente Responsable.

Si aplicáramos estrictamente el criterio de Baron, reduciríamos mucho el número de empresas que se pueden considerar Socialmente Responsables. La razón es que hay muchas estrategias empresariales que pueden ser consideradas como Socialmente Responsables porque serían implementadas por empresas altruistas y que, a su vez, no entran en contradicción con el criterio maximizador de la empresa neoclásica. Incluso se podría ser más provocador, preguntándose si existe alguna empresa cuyo comportamiento pasa el filtro de Baron y se puede realmente considerar que presenta un comportamiento Socialmente Responsable.

Nuestra opinión, sin embargo, difiere de la de Baron (2001). La RSC, desde nuestro punto de vista, tiene que ver con una estrategia de cooperación con los dis- 
tintos stakeholders de la empresa (por ejemplo, responder a una demanda de los consumidores) y con una visión a largo plazo en la maximización de los beneficios empresariales. Esto implica, por lo tanto, que la RSC no está en contradicción con la rentabilidad empresarial, más bien al contrario Así, a continuación vamos a enumerar algunas estrategias empresariales Socialmente Responsables que no están en conflicto con una estrategia de maximización de beneficios de una empresa tradicional o neoclásica.

Internalizar las externalidades para eludir un potencial boicot de los consumidores o para obtener fondos de inversión Socialmente Responsables.

En el modelo de la sección anterior, hemos analizado en detalle como el miedo al boicot de los consumidores activistas puede generar un comportamiento altruista en una empresa cuyo único objetivo es maximizar beneficios. El comportamiento social de la empresa, puede afectar además de la demanda, a las fuentes de financiación de la empresa. De acuerdo con el artículo de Lafuente et al (2003), existen instituciones de inversión colectiva e inversores particulares que tienen en cuenta el comportamiento social de las empresas para tomar sus decisiones de inversión. Estos fondos «éticos», se comportan como los consumidores activistas, restringen sus inversiones a aquellas que consideran Inversión Socialmente Responsable, y pueden discriminar a empresas por utilizar trabajo infantil o tener activos en países que no respetan los derechos humanos. Por ejemplo, según Lafuente et al (2003) los primeros fondos de inversión colectiva que realizaron boicot a determinadas empresas, eran fondos con motivaciones religiosas (cuáqueros) en los años cincuenta, estos fondos no invertían en los denominados «valores pecados», acciones de empresas cuyo negocio estaba relacionado con el alcohol, el juego y el tabaco. El comportamiento de estos fondos religiosos pone de manifiesto que no siempre puede existir consenso sobre lo que es un comportamiento socialmente responsable. Comentaremos esta cuestión más adelante. Por último, de la misma forma que los consumidores activistas penalizan un comportamiento oportunista con una reducción de demanda, también un comportamiento socialmente positivo de la empresa puede ser recompensado con un aumento de la demanda. Esto podría explicar las actividades de patronazgo y las subvenciones/ayudas empresariales a actividades culturales y sociales.

La externalidad producida por la empresa afecta al valor del bien para los consumidores

Este es el caso discutido en la introducción. Un hotel se preocupa de no estropear la playa más cercana, porque el estado de esa playa afecta a la valoración que tienen los consumidores del hotel en si mismo. El control de la externalidad determi- 
na la calidad percibida del servicio. Reducir el efecto externo implica para la empresa apostar por calidad alta, estrategia de mercado que puede ser perfectamente consistente con un comportamiento de maximización de beneficio.

Comportamiento altruista hacia los consumidores, por consideraciones dinámicas de imagen de la marca

Los contratos entre los consumidores y las empresas son necesariamente incompletos porque, por ejemplo, los consumidores no pueden evaluar la calidad de los productos hasta que los consumen. Esto en última instancia, puede tener como consecuencia que desaparezca el mercado de los productos de calidad alta. El argumento desarrollado por primera vez por Akerlof (1970) y conocido como el problema de selección adversa es el siguiente: Dado que como los consumidores no pueden verificar la calidad, la estrategia de la empresa es anunciar que la calidad es alta, pero minimizar los costes produciendo calidad baja. Los consumidores anticipan este comportamiento y solo están dispuestos a pagar el valor del bien de calidad baja. En consecuencia solo se produce calidad baja. Para escapar de esta situación que es claramente ineficiente, se pueden introducir salvaguardas contractuales como garantías ${ }^{6}$, o establecer una reputación en el mercado de calidad alta, a través de lo que se denomina un contrato implícito. Los consumidores confían en la empresa (marca) que tiene una reputación de calidad alta, porque saben que si produce calidad baja, perderá la reputación (la confianza) de los consumidores y como consecuencia de ello, unos grandes beneficios en el futuro. Este mecanismo de reputación lleva a las empresas no solo a producir calidad alta, sino a salvaguardar su imagen de marca, ofreciendo servicios post-venta, más allá de sus compromisos contractuales. De nuevo, este comportamiento mirado desde una perspectiva estática se puede confundir como «altruista», pero es perfectamente consistente con la maximización de beneficios desde una perspectiva dinámica. La idea de que relación dinámica con los consumidores daba incentivos a la empresa para eludir el oportunismo, fue desarrollada en primer lugar por Klein y Leffler (1991). Por ejemplo, recientemente la empresa Volvo ha decidido cambiar gratuitamente los radiadores de un modelo de lujo, porque una parte de los automóviles de un lote de producción, ha aparecido una deficiencia. Contractualmente, Volvo, no estaría obligado a ello, en una parte de los coches no tendrán ninguna anomalía y otra parte de los mismos, la tendrán cuando haya expirado la garantía. Así, parece claro como este comportamiento socialmente responsable de Volvo está originado con el fin de salvaguardar su imagen de marca, y mantener el compromiso de calidad alta con los consumidores.

${ }^{6}$ Las garantías sirven para resolver el problema, porque se pueden dar equilibrios en los que solamente las empresas que producen calidad alta ofrecen garantías. En este caso la existencia de garantía señaliza calidad alta porque para las empresas de calidad baja tiene un coste excesivo garantizar el producto. 
Comportamiento Socialmente Responsable con el objetivo estratégico de evitar la respuesta reguladora

La idea es que puede haber empresas que internalicen parcialmente las externalidades que producen, para evitar que surjan grupos de interés que presionen para que haya una respuesta reguladora. Por ejemplo, durante mucho tiempo Microsoft se podía considerar un monopolista tanto en el mercado de sistemas operativos, como en el mercado de software básico para oficina. Sin embargo Microsoft cargaba un precio que todos los especialistas consideraban muy inferior al del monopolista. Una posible explicación de este comportamiento es que con ello quería evitar que las autoridades de defensa de la competencia intervinieran, como luego finalmente sucedió. El argumento es muy similar al que se utiliza en Economía Industrial para estudiar los problemas de entrada a un mercado. En este contexto, la teoría de los precios límite dice que un monopolista puede poner precios más bajos para prevenir la entrada de nuevos competidores, señalizando a través de los precios la poca rentabilidad futura del mercado en competencia; ver por ejemplo Milgrom y Roberts (1984).

\section{Comportamiento altruista (cooperativo) con proveedores y trabajadores}

Existen empresas que tienen un comportamiento muy cooperativo tanto con proveedores como con los trabajadores. Por ejemplo, hay empresas, que ayudan a sus proveedores a financiar los cambios de tecnología, que discuten con ellos, los posibles cambios de diseño del producto o incluso que se les paga un cierto sobreprecio por los insumos. De igual forma, hay empresas, que pagan salarios mejores que los del sector, que invierten en la formación generalista de sus trabajadores, que tienen una política de no despidos, y mejoran de forma sistemática los programas de bienestar y apoyo a los trabajadores. Estas políticas, de nuevo pueden justificarse dentro del paradigma neoclásico de la maximización de beneficios. El argumento es muy similar al del segundo punto, y se conoce en la literatura como los contratos relacionales (ver Baker et al., 1994, y Bull, 1987) y como la teoría de los salarios de eficiencia (Shapiro y Stigligtz, 1984). La idea es la siguiente: debido a las imperfecciones de los contratos las relaciones contractuales entre el proveedor y la empresa están llenas de situaciones en las cuales el proveedor se puede comportar de forma oportunista. Por ejemplo: un cambio tecnológico reduce el coste del insumo. Esto hace necesario la reducción del precio del insumo para mantener la competitividad de la empresa, y el proveedor se puede negar a ello, para extraer una mayor parte del excedente. Para inducir un comportamiento cooperativo del proveedor, lo que hace la empresa es darle al proveedor una renta adicional (comportarse de forma altruista con él). Dado este comportamiento, el proveedor renunciará a comportarse de forma oportunista en el corto plazo, por el temor a perder en el largo plazo esta renta adicional a la que obtendría en el mercado. La teoría de los salarios de efi- 
ciencia, desarrolla el mismo argumento para justificar pagar salarios por encima de las productividades de los trabajadores. Los trabajadores tienen incentivos a esforzarse y comportarse de acuerdo con los intereses de la empresa, porque si no lo hacen corren el riesgo de ser despedidos, y perder la renta adicional. ${ }^{7}$

En resumen, el uso de estas estrategias a menudo denominadas socialmente responsables no resulta incompatible con la hipótesis de maximización de beneficios. Un caso especial son las empresas que no tienen como objetivo único la maximización de beneficios (o al menos no de forma estricta) como las Organizaciones No Gubernamentales, algunas empresas públicas, o entidades gobernadas por stakeholders (organismos sociales) como las Cajas de Ahorro. Sin embargo, aunque estas organizaciones están gobernadas, al menos indirectamente, por algunos stakeholders, no significa que necesariamente no tengan conflictos con otros grupos de stakeholders. Por ejemplo, una ONG dedicada a la ayuda humanitaria podría presentar algún conflicto de Responsabilidad Social con organizaciones defensoras del medioambiente, o con sus propios trabajadores.

\section{RSC, regulación, activismo y bienestar}

A lo largo del texto estamos identificando el comportamiento poco responsable con la generación de una externalidad negativa. Tradicionalmente la forma que tiene la teoría económica de resolver los problemas relacionados con las externalidades es a través de la regulación o de impuestos pigovianos. Nos podemos entonces preguntar, ¿porqué no resolver el problema con estos instrumentos?, ¿Porqué no obligar a las empresas a través de la regulación a ser socialmente responsables?, ¿qué ventajas, si éstas existen, tiene la auto-regulación del mercado sobre una regulación pública formal?

Efectivamente, en condiciones ideales ((i) información perfecta sobre el comportamiento de la empresa y las preferencias de los consumidores sobre ese comportamiento, (ii) capacidad de compromiso, y (iii) costes de transacción bajos de implementación y control), la regulación es un instrumento que sirve para que las empresas internalicen las externalidades y se maximice el bienestar social. ${ }^{8}$ Por ejemplo, en nuestro problema bastaría con imponer a la empresa una multa/impuesto B cuando utiliza la tecnología sucia para que ésta tuviera incentivos a cambiar de tecnología, maximizando el bienestar social.

Sin embargo, existen múltiples contextos en los que la regulación no puede implementar la asignación óptima. Sin pretensión de exhaustividad, y de forma meramente intuitiva, proponemos tres posibles contextos/situaciones en las que la regulación gubernamental puede no ser eficaz y, en cambio, los incentivos del mer-

\footnotetext{
${ }^{7}$ En la misma línea, KREPS (1990) utiliza un argumento dinámico similar, para justificar la existencia de culturas cooperativas en las empresas.

${ }^{8}$ Para un análisis detallado de la teoría de la regulación, ver LAFFONT y TIROLE (1996).
} 
cado (vía activismo y amenazas de boicot de parte de los consumidores) pueden inducir a las empresas a comportarse de forma responsable y mejorar el bienestar social.

En el modelo desarrollado más arriba hemos supuesto que los consumidores activistas conocían el tipo de tecnología adoptada por la empresa. Entonces, por consistencia suponemos también que el regulador puede tener acceso a la misma información que los consumidores. Sin embargo, el regulador se enfrenta a una restricción adicional: la regulación debe estar basada en información verificable, que pueda ser llevada y defendida ante un tribunal. Supongamos que el regulador no puede tener acceso a información verificable ante un tribunal respecto a la tecnología que esté siendo usada por la empresa, ni tampoco información verificable respecto a si efectivamente se ha generado la externalidad. En tal caso, el regulador difícilmente podrá implementar la solución óptima respecto a la adopción de la tecnología. Sin embargo, el activismo de parte de los consumidores (mediante la amenaza de boicots) no se enfrenta a esta restricción. Es suficiente con disponer de la información para, mediante amenaza de boicot de los consumidores activistas, inducir en la empresa un comportamiento socialmente responsable.

Puede que para algunas empresas sea más barato que para otras reducir la externalidad. Con información perfecta, esto llevaría a la existencia de diferentes estándares de regulación en función de la tecnología de la empresa (la regulación óptima consistiría en exigir a la empresa eficiente que adoptase una tecnología más limpia a cambio de una mayor compensación). Sin embargo, debido a restricciones en la información verificable disponible, lo cual puede implicar en algunos casos la imposibilidad de poder discriminar entre las empresas, la Administración regula de forma homogénea exigiendo el mismo estándar a todas las empresas. En este caso, la autorregulación inducida por la presión de los consumidores activistas (no restringidos por la necesidad de disponer de información verificable\}) podría jugar un papel positivo induciendo a las empresas más eficientes a ir mas allá de los estándares fijados por la regulación.

Finalmente, otra posibilidad por la cuál el comportamiento responsable de las empresas puede venir dado por las presiones del mercado, y no por la regulación, está basada en la posible captura del regulador por parte de las empresas del sector; la captura del regulador significa que, mediante distintos mecanismos, las empresas disponen de la suficiente capacidad para influir en su propia regulación. En tal caso de captura del regulador, la regulación será favorable para las empresas del sector y, previsiblemente, no inducirá al comportamiento socialmente eficiente de las empresas. En este contexto, la presión del mercado (mediante activismo, boicots) podría jugar un papel positivo induciendo a las empresas a adoptar un comportamiento socialmente responsable.

Resumiendo, desde nuestra perspectiva los estándares de comportamiento de la RSC juegan un papel suplementario respecto la regulación gubernamental. Así, en aquellos contextos en los que la intervención gubernamental se ve limitada por algún factor (información, contratos incompletos, etc.), la presión de mercado vía activis- 
mo de consumidores u organizaciones interpuestas (ONGs) podría suplir esta ausencia de la regulación. Así, en la siguiente subsección, a partir del modelo expuesto más arriba, llevamos a cabo un análisis de bienestar, y un análisis del papel y de las características del activismo como mecanismo inductor de la RSC. Sin embargo, tal y como explicamos más abajo, es importante señalar que incluso en este contexto de auto-regulación de las empresas vía activismo de los consumidores, el sector público debe desempeñar un papel relevante en la generación de la información de que puedan disponer los consumidores.

\section{Análisis de bienestar}

Un paso previo a hacer un mínimo análisis de bienestar sobre el modelo de la sección anterior es justificar las preferencias de los consumidores no activistas. Cuando asumimos que los consumidores no activistas no tienen en cuenta la externalidad en sus decisiones de consumo, comportamiento que modelizamos a través de la función de utilidad,

$$
u_{N A}=v-p
$$

en realidad no estamos diciendo que necesariamente a los consumidores no les importa la externalidad. A este grupo de consumidores les puede importar la externalidad pero, sin embargo, no tenerla en cuenta. Existen dos posibles explicaciones. Una primera explicación está basada en que el bien consumido sea un bien de confianza (credence good; ver Tirole, 1991, capítulo 2), lo cual significa que el consumidor no dispone de información respecto a si efectivamente se ha generado una externalidad (esta posibilidad es especialmente razonable en el contexto de nuestro modelo en el que la externalidad se produce en el proceso de producción). Una segunda explicación es que, incluso en el caso de que el consumidor dispusiera de información respecto a la existencia de la externalidad, se comportara de forma oportunista. El consumidor piensa que el efecto de su decisión de compra sobre el nivel total de externalidad que va a asumir es minúsculo y, por lo tanto, ignora este efecto. Su comportamiento es similar al descrito, por ejemplo, en teoría de juegos en el dilema de los presos. Cada consumidor no activista preferiría que ningún consumidor no activista comprase bienes producidos con tecnología sucia a que todos lo comprasen. Pero su bienestar se maximiza cuando todos los demás no compran y él lo hace. Es lo que se denomina también el problema del pasajero sin billete (o free-rider).

Por todo esto, y de cara a hacer un análisis de bienestar, vamos a suponer que aunque no lo tienen en cuenta, los consumidores no activistas en principio también pueden obtener un perjuicio por la externalidad negativa; podemos considerar que el perjuicio puede ser más pequeño que el de los consumidores activistas, $\theta \cdot \mathrm{B}$, donde $0 \leq \theta \leq 1$ En realidad, $\theta$ va a depender mucho de la externalidad o comportamiento social que estemos considerando. Hay externalidades qu podemos denominar 
«globales», por que pueden afectar a casi todo el mundo por igual. Consideremos por ejemplo, comportamientos de la empresa que afectan al medioambiente de forma «global», como calentamiento de la atmósfera o deforestación de la selva amazónica, en estos casos 1 debe ser muy alto. Sin embargo, también existen externalidades que podemos denominar «privadas» que sólo afectan a grupos y no tienen ningún impacto sobre el resto de la sociedad. Por ejemplo, un grupo religioso puede ser un consumidor activista contra una empresa que reinvierte parte de sus beneficios en un canal erótico para adultos. Los consumidores no activistas en este caso puede que consideren que esta actividad no tiene un efecto negativo sobre ellos $(\theta=0) \mathrm{y}$, en este caso, su comportamiento no será oportunista, sencillamente no internalizarán la externalidad en su decisión de compra por que para ellos no existe.

Consideremos por ahora que estamos ante una externalidad global y que $\theta=1$. Por lo tanto, desde el punto de vista social, el bienestar que se produce con la tecnología limpia es

$$
w(L)=v-c_{A}>0
$$

mientras que el que se produce con la tecnología sucia es (solamente los consumidores no activistas compran y consumen el bien

$$
w(S)=(1-\alpha)\left(v-c_{B}-B\right)<0
$$

Está claro que, desde el punto de vista del bienestar, lo mejor es utilizar la tecnología limpia. De la sección anterior sabemos que si la empresa maximiza sus beneficios solamente utilizará esta tecnología limpia en el caso en que $\alpha>\frac{c_{A}-c_{B}}{v}$. Desde el punto de visto de los consumidores no activistas, la existencia de los consumidores activistas tienen un efecto positivo ya que (estamos suponiendo que) aunque no la tomen en cuenta en su decisión de compra, sí la sufren.

Sin embargo, esto no tiene que ser el caso con las externalidades privadas. En este caso, la existencia de consumidores activistas puede reducir el bienestar del resto de los consumidores. La intuición es sencilla; imaginemos que un grupo religioso puede organizar boicots contra periódicos que incluyen anuncios relacionados con el sexo; al resto de los lectores del periódico la existencia de este tipo de anuncios no les supone ninguna molestia. Puede que la influencia de estos consumidores activistas consiga que el periódico suprima este tipo de anuncios, con el efecto colateral de que la reducción de ingresos genere un incremento en el precio del periódico. De ser así, habrá habido una redistribución de bienestar, con consecuencias positivas para el grupo activista y negativas para el resto. En resumen, desde el punto de vista del bienestar los consumidores activistas pueden tener un efecto positivo sobre el resto de los consumidores cuando se trata de externalidades globales. Cuando se trata de externalidades privadas el efecto de los consumidores activistas no es necesariamente positivo sobre el resto de los grupos de consumidores. 


\section{Activismo e información pública}

Dado que la presencia de consumidores activistas tiene efectos importantes sobre el bienestar, porque alteran los incentivos y el comportamiento de las empresas, es interesante profundizar en sus motivaciones y los patrones que determinan su presencia y proporción en el conjunto de consumidores. Para que un consumidor actúe como consumidor activista debe reunir dos condiciones:

1. Debe ser altruista;

2. Debe tener información sobre la existencia o no de una externalidad.

El activismo de los consumidores activistas implica que son capaces de anteponer a su decisión de consumo, consideraciones de tipo social. Este altruismo es el factor que elimina en estos consumidores el problema del pasajero sin billete (o freerider) explicado más arriba, y hace que los consumidores no presenten un comportamiento oportunista.

El otro aspecto clave en el activismo de los consumidores es la información. Los consumidores, además de ser altruistas y, por lo tanto, no oportunistas, deben de tener la información adecuada respecto a las consecuencias de la acción empresarial. Deben de saber cuando efectivamente se genera una externalidad y también la magnitud de esta externalidad.

Esto nos lleva a la conclusión de que generar información sobre el comportamiento de las empresas es positivo (al menos con respecto a las externalidades globales) porque permite la existencia de consumidores activistas que finalmente disciplinarán a las empresas. En la generación de información pueden intervenir diversos actores. Por un lado, las organizaciones de stakeholders, como podemos denominar a las asociaciones de afectados por la actividad de las empresas (asociaciones en defensa del medioambiente, de los derechos humanos, etc.). Estas organizaciones actúan como un grupo de presión («lobby») y gran parte de su actividad consiste en generar información. Otro actor importante son las propias empresas, que tienen interés en publicitar su comportamiento social cuando éste sea considerado «positivo», y tienen interés en contrarrestar la información proveniente de las organizaciones de stakeholders cuando éstas consideran que la actividad de la empresa es negativa.

El último actor implicado es el sector público. Debido a que la información en si misma es un bien público, sabemos que los incentivos privados no aseguran que se provea de forma eficiente. Aunque la existencia de altruismo y de conflicto de intereses, puede mitigar la infra producción de información, en general se puede suponer que el sector público puede aumentar el bienestar aportando información sobre el comportamiento de las empresas. En primer lugar, puede regular la provisión de información de las partes implicadas, generando reglas de transparencia sobre el comportamiento de las empresas, y sistemas de verificación de la información. Y en segundo lugar, el sector público puede hacer una labor importante seleccionando la 
información. Aunque nos estamos refiriendo a la información e incluso a la externalidad como si fueran fenómenos unidimensionales, existen muchos tipos distintos de comportamiento social, y existe una información muy dispersa sobre el comportamiento de las empresas (las empresas, por ejemplo, actúan en diversos ámbitos, el autonómico, nacional, europeo, y en cada uno de ellos se provee información). Existen pues multitud de medidas, y esto no siempre significa más y mejor información. Generar o seleccionar un número reducido de índices, basados en medidas de cumplimientos de estándares fiables, puede mejorar la información que reciben los consumidores y, con ello, los incentivos de las empresas a un comportamiento socialmente responsable.

Finalmente, añadir que dejamos a un lado el debate respecto a si esta actividad del sector público puede ser desarrollada por intermediarios privados. En este sentido debemos mencionar que existe una literatura importante sobre el papel de los intermediarios en los mercados de información incompleta. El principal resultado de esta literatura es que la existencia de estos intermediarios generadores de información aumentan el bienestar de los participantes en el mercado. Los mercados de expertos son un buen ejemplo de los bienes de confianza (como hemos dicho, bienes en los que los consumidores no conocen la calidad del bien, ni siquiera después de haberlo consumido.): uno no puede valorar completamente la calidad de los servicios de un abogado o un médico justo después de haberlos recibido. Wolinsky (1993), analiza este tipo de mercados y caracteriza los equilibrios, poniendo de manifiesto las ineficiencias que la falta de información puede generar. Taylor (1995) y Lizzeri (1999) demuestran que la existencia de intermediarios informados que aminoren la asimetría de información entre oferta y demanda, puede aumentar el excedente global. En el caso de la RSC el papel del intermediario informado está asociado en muchas ocasiones a la intervención de asociaciones independientes, como por ejemplo, los índices de bolsa de empresas socialmente responsables (Dow Jones Sustainability Index, FTSE4Good), o los estándares de provisión de información empresarial como el Global Reporting Indez (GRI). Esto, claro, no excluye el papel que el sector público puede jugar como, por ejemplo, con la aprobación de normativas respecto a la información que las empresas deben aportar a los consumidores (etiqueta energética, por ejemplo).

\section{Conclusiones}

En este artículo se ha presentado un análisis de la Responsabilidad Social Corporativa de las empresas desde el punto de vista de la Teoría Económica. Por empresa Socialmente Responsable se entiende normalmente a aquella empresa que toma sus decisiones teniendo en consideración los intereses de todos los stakeholders de la empresa (todos aquellos afectados por las acciones de la empresa), y no solamente los intereses de los accionistas. Desde nuestra perspectiva, la RSC está originada en una demanda del mercado (por ejemplo, en una demanda de algunos consumidores) 
y sencillamente consiste en primar una actitud de cooperación con los distintos stakeholders de la empresa y adoptar una visión de la maximización de la rentabilidad empresarial a largo plazo. Con un modelo sencillo demostramos que, dado que existen consumidores activistas que penalizan los comportamientos socialmente no responsables de las empresas, éstas pueden tener incentivos (incluso bajo la hipótesis de maximización de beneficios) a comportarse como una empresa socialmente responsable. Este modelo sencillo nos permite transmitir esta idea que la RSC responde a una demanda del mercado, aunque ésta en la realidad puede venir dada por distintos mecanismos, tal y como también explicamos en el artículo.

A continuación, en el artículo se analiza el papel de la RSC con respecto a la regulación tradicional. Nuestro punto de vista es que la RSC y la regulación son sustitutivos, por lo que la RSC adquiere más importancia en entornos donde por alguna razón la regulación no es efectiva. Realizando un análisis muy sencillo de bienestar se muestra además que los consumidores activistas' pueden tener un efecto positivo sobre el resto de consumidores. Este hecho justifica que el sector público apoye la existencia de esta parte de la demanda sensible al comportamiento social de la empresa, fundamentalmente proveyendo información sobre el comportamiento social de las empresas, además de dando instrumentos para que la información generada por otros agentes sea fiable y verificable por los consumidores.

\section{Referencias}

[1] AKERLOF, G. (1970). «The Market for "lemons": Quality Uncertainty and the Market Mechanism». Quarterly Journal of Economics, 84, 488-500.

[2] ALLEN, F. y GALE, D. (2000). «Corporate governance and competition: theoretical and empirical perspectives», en VIVES, C. (ed.), Corporate governance: theoretical and empirical perspectives, Cambridge University Press, Cambridge, págs 23-94.

[3] BAKER, G, R. GIBBONS y K. MURPHY (1994). «Subjective Performance Measures in Optimal Incentive Contracts», Quarterly Journal of Economics, 109, 1125-56.

[4] BARON, D. (2001). «Private Politics, Corporate Social Responsibility, and Integrated Strategy», Journal of Economics \& Management Strategy, 10, 1, 745-764.

[5] BULL, C. (1987). «The Existence of Self-Enforcing Relational Contracts», Quarterly Journal of Economics, 102, 147-59.

[6] CESPA, G. y CESTONE, G. (2002). «Stakeholder Activism, Managerial Entrenchment, and the Congruence of Interests Between Small Shareholders and Stakeholders». UPF Working Paper, 634.

[7] CRESPÍ, R.; JANSSON, E. y SALAS, V. (1998). «La valoración económica de las cajas de ahorros». Papeles de Economía Española, 74-75, 109-126.

[8] FEDDERSEN, T. y GILLIGAN, T. (2001). «Saints and Markets: Activists and the Supply of Credence Goods». Journal of Economics \& Management Strategy, 10.

[9] HANSMANN, H. (1996). The ownership of enterprise. Cambridge, MA:

[10] HOLMSTROM, B. y P. MILGROM (1991). «Multi-Task Principal-Agent Problems: Incentive Contracts, Asset Ownership, and Job Design». Journal of Law, Economics and Organization, 7, 24-52. 
[11] KLEIN, B. y LEFFLER, K. (1981). «The role of market forces in assuring contractual performance», Journal of Political Economy, 89, 615-641.

[12] KREPS, D. (1990). «Corporate Culture and Economic Theory», Perspectives on Positive Political Economy, editado por James E. Alt y Kenneth A. Shepsle, Cambridge University Press.

[13] LAFFONT, J.-J. y J. TIROLE (1993). A Theory of Incentives in Procurement and Regulation, Cambridge: MIT Press.

[14] LIZZERI, A. (1999). «Information Revelation and Certification Intermediaries», Rand Journal of Economics, 30, 214-231.

[15] LAFUENTE, A.; VIÑUALES, V.; PUEYO, R. y LLARÍA, J. (2003). «Responsabilidad Social Corporativa y Políticas Públicas». Documento de Trabajo 3/2003.

[16] RODRÍGUEZ (2002). «Teoría de los partícipes y ciudadanía empresarial: una perspectiva pluralista del gobierno de las compañías». Ekonomiaz, 50, 2. ${ }^{\circ}$ cuatrimestre.

[17] SHAPIRO, C y J. STIGLITZ (1984). «Equilibrium Unemployment as a Discipline Device», American Economic Review, 74, 433-44.

[18] TAYLOR, C. (1995). «The Economics of Breakdowns, Checkups, and Cures». Journal of Political Economy, 103, 53-74.

[19] TIROLE, J. (1990). La teoría de la organización industrial. Ed. Ariel Economía.

[20] WOLINSKY, A. (1993). «Competition in a Market for Informed Experts' Services». Rand Journal of Economics, 24, 380-398. 